\title{
Iron oxide nanoparticles for plant nutrition? A preliminary Mössbauer study
}

\author{
Z. Homonnay ${ }^{1}$, Gy. Tolnai ${ }^{2}$, F. Fodor ${ }^{3}$, Á. Solti $^{3}$, K. Kovács ${ }^{1}$, E. Kuzmann ${ }^{1}$, \\ A. Ábrahám ${ }^{1,2}$, E.Gy. Szabó ${ }^{2}$, P. Németh ${ }^{2}$, L. Szabó ${ }^{2}$, Z.Klencsár ${ }^{2}$ \\ ${ }^{1}$ Institute of Chemistry, EötvösLoránd University, 1117Pázmány P. s. 1/A, \\ Budapest, Hungary \\ ${ }^{2}$ Institute of Materials and Environmental Chemistry, Research Centre for Natural \\ Sciences, HAS, Magyar tudósokkörútja 2, Budapest, Hungary \\ ${ }^{3}$ Institute of Biology, EötvösLoránd University, 1117Pázmány P. s. 1/C, Budapest, \\ Hungary \\ E-mail addressofthe corresponding author: homonnay@caesar.elte.hu
}

\begin{abstract}
One of the most important micronutrients for plants is iron. We have prepared iron(III) oxyhydroxide and magnetite nanoparticles with the aim to use them as possible nutrition source for plants. The iron(III)-oxide/oxyhydroxide nanoparticles prepared under our experimental conditions as colloidal suspensions proved to be 6-line ferrihydritenanoparticles as verified by XRD,TEM/SAED and Mössbauer spectroscopy measurements. ${ }^{57} \mathrm{Fe}$ Mössbauer spectra of magnetite nanoparticles prepared under different preparation conditions could be analyzed on the basis of a common model based on the superposition of four sextet components displaying Gaussian-shaped hyperfine magnetic field distributions.
\end{abstract}

Keywords:plant nutrition, ${ }^{57} \mathrm{Fe}$ Mössbauer spectroscopy, magnetite, ferrihydrite, nanoparticles 


\section{Introduction}

As the World's population keeps increasing, agriculture is getting more and more into the focus of interest of research. To alleviate problems related to population growth and associated shortage of food resources, the increase of food production efficiency is a key question. Its success highly depends on how it is possible to grow healthy plants. For high quality crop and efficient production, ensuring optimal nutrition for the plants is a necessity: in case of low-quality soil (such as those with high soil $\mathrm{pH}$ ), missing nutrients should be supplied by appropriate fertilizers. Iron is one of the most important micronutrients for plants: insufficient supply of iron seriously hampers chlorophyll biosynthesis in the leaves, resulting in slow growth, diminishing plant biomass and nutrition value (Fig. 1). Decreased availability or disturbed uptake of iron may also lead to the death of the plants.
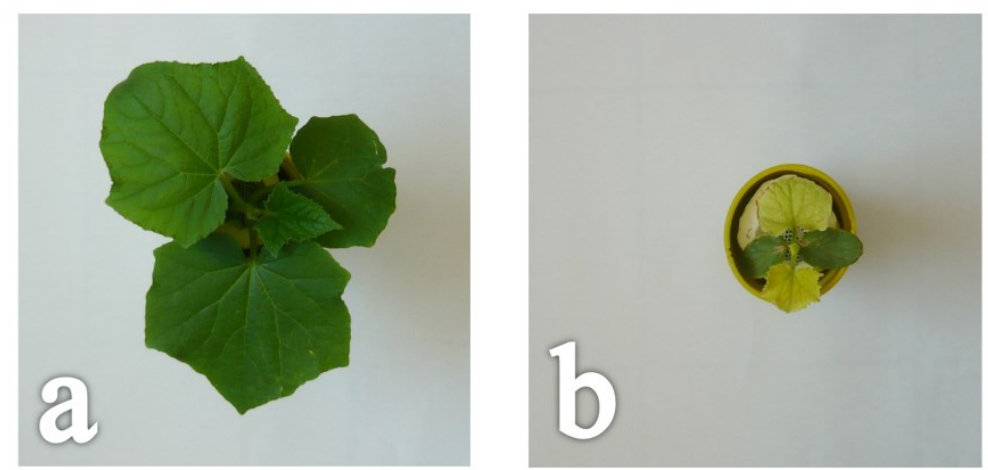

Figure 1. Illustration of the effect of insufficient iron supply on the development of cucumber plant: a, plant grown under normal conditions, b, plant grown under conditions with insufficient iron supply.

Uptake of iron by plants from the soil is known to proceed by two different strategies which have been previously studied by us using Mössbauer spectroscopy [1,2]. These strategies assume dissolved iron(III) that either forms a complex to be able to get through the cell membranes of the root, or is first reduced to iron(II) and then transferred into the cytoplasm in this form. Since storage of iron in cells is usually realized in the form of ferritin whose core bears similarities with a ferrihydrite/ferric hydrous oxide nanoparticle with variable amounts of phosphate [3], one may consider plant nutrition supply in the soil directly by iron-based oxide or oxyhydroxide nanoparticles. It may be mentioned that some ferritin analogues are used for treatment of iron deficiency anemia. 
In our research, we have prepared iron(III)-oxyhydroxide and magnetite/maghemite nanoparticles with the aim to use them as possible nutrition source for plants, and along with other methods, we also characterized them by ${ }^{57} \mathrm{Fe}$ Mössbauer spectroscopy. The first plant growth experiments indicate successful iron uptake from colloidal suspensions of ferrihydrite nanoparticles. The mechanism of the uptake is a subject of ongoing studies.

\section{Experimental}

Stable colloidal suspension ( $\mathrm{pH} \approx 2.2$ ) of iron(III)-oxyhydroxide nanoparticles was prepared via wet chemical synthesis with a concentration of $\sim 0.37 \mathrm{mg} / \mathrm{cm}^{3}$ for the colloidal fractions (sample NS1). The corresponding iron concentration was estimated to be $\sim 0.26 \mathrm{mg} / \mathrm{cm}^{3}$. An associated colloidal suspension was prepared and dried to yield an analogous sample in the powder form (sample NS2).

$\mathrm{Fe}_{3} \mathrm{O}_{4}$ nanopowders were prepared via chemical co-precipitation method starting with the preparation of a mixed solution of $\mathrm{Fe}^{2+}$ and $\mathrm{Fe}^{3+}$ salts, and then reacting it with a strongly alkaline aqueous medium. After the reaction was completed, the magnetite particles were filtered, washed several times with distilled water, and then dried. In order to investigate the effect of preparation conditions on the oxidation state of iron in the resulting nanoparticles, the samples were

- prepared under either ambient conditions or nitrogen atmosphere,

- prepared with or without a coating agent (malic acid), and

- dried either at room temperature or at $80^{\circ} \mathrm{C}$.

Here we focus our attention on the Mössbauer study of two of the samples that were found to represent extreme cases (among those prepared) from the point of view of the oxidation of iron in the corresponding nanoparticles. On the one hand, we discuss the case of sample M2 that was prepared under ambient atmosphere without malic acid, and was dried at $80^{\circ} \mathrm{C}$. On the other hand, we present results concerning sample M4 that was prepared under ambient atmosphere with malic acid applied and dried at room temperature. A further detailed description of the synthesis of the samples will be given elsewhere. 
Morphology of the samples was studied by the means of transmission electron microscopy (TEM) measurements carried out by a Morgagni 268D (operating at $100 \mathrm{kV}$ ) instrument.

Phase purity and crystal structure of the prepared samples were checked at room temperature by powder X-ray diffractometry by using $\mathrm{CuK} \alpha$ radiation in a Philips PW3710 based PW1050 Bragg-Brentano parafocusing goniometer system with graphite monochromator and a proportional counter.

${ }^{57} \mathrm{Fe}$ Mössbauer spectroscopy measurements were performed with WISSEL spectrometers applied in transmission geometry either at room temperature or at low temperature in liquid nitrogen cooled bath type cryostats. ${ }^{57} \mathrm{Co}$ in $\mathrm{Rh}$ matrix served as a source of $20 \mathrm{mCi}$ activity. The isomer shifts are given relative to $\alpha-\mathrm{Fe}$ at room temperature. The Mössbauer spectra were evaluated via least-squares fitting by using version 4.0Pre of the MossWinn program [4].

\section{Results and discussion}

TEM measurements (Fig. 2) confirmed that samples NS1 and NS2 are both composed of nanoparticles. The latter, powdered sample clearly shows agglomeration of closely attached nanoparticles, whereas the similarly sized $(\sim 5 \mathrm{~nm})$ quite monodisperse particles are only loosely bound in the colloidal suspension. Selected area diffraction patterns (overlaid on the corresponding TEM images in Fig. 2) reveal similar diffraction patterns in the two cases corroborating that the nanoparticles have closely related, possibly identical crystal structures in the two samples. The X-ray diffractogram of sample NS2 (Fig. 3) confirmed that the crystal structure in question is that of 6-line ferrihydrite [5].

The ${ }^{57} \mathrm{Fe}$ Mössbauer spectrum of NS2 at room temperature (Fig. 4) can be decomposed into two Lorentzian doublets with parameters (Table 1) and a spectral area ratio $(\sim 2: 1)$ quite close to the corresponding parameters of $\beta-\mathrm{FeOOH}[6]$. This suggests that the local microenvironments of iron in our nanoparticles are quite similar to those in akaganéite. Nevertheless, one should keep in mind that paramagnetic ferric oxyhydroxides or hydrous oxides all have very similar Mössbauer parameters. 
Table $1{ }^{57} \mathrm{Fe}$ Mössbauer parameters of the doublets contributing to the spectrum fit of sample NS2 at room temperature. Numbers in parentheses denote the statistical uncertainty (1 sigma) in the last digit of the corresponding values.

\begin{tabular}{|l|l|}
\hline Doublet (I) & $68(4) \%$ \\
\hline$\delta, \mathrm{mm} / \mathrm{s}$ & $0.368(1)$ \\
\hline$\Delta, \mathrm{mm} / \mathrm{s}$ & $0.53(1)$ \\
\hline$\Gamma, \mathrm{mm} / \mathrm{s}$ & $0.40(1)$ \\
\hline & \\
\hline Doublet (II) & $32(4) \%$ \\
\hline$\delta, \mathrm{mm} / \mathrm{s}$ & $0.349(2) \mathrm{mm} / \mathrm{s}$ \\
\hline$\Delta, \mathrm{mm} / \mathrm{s}$ & $0.91(2) \mathrm{mm} / \mathrm{s}$ \\
\hline$\Gamma, \mathrm{mm} / \mathrm{s}$ & $0.39(1) \mathrm{mm} / \mathrm{s}$ \\
\hline
\end{tabular}
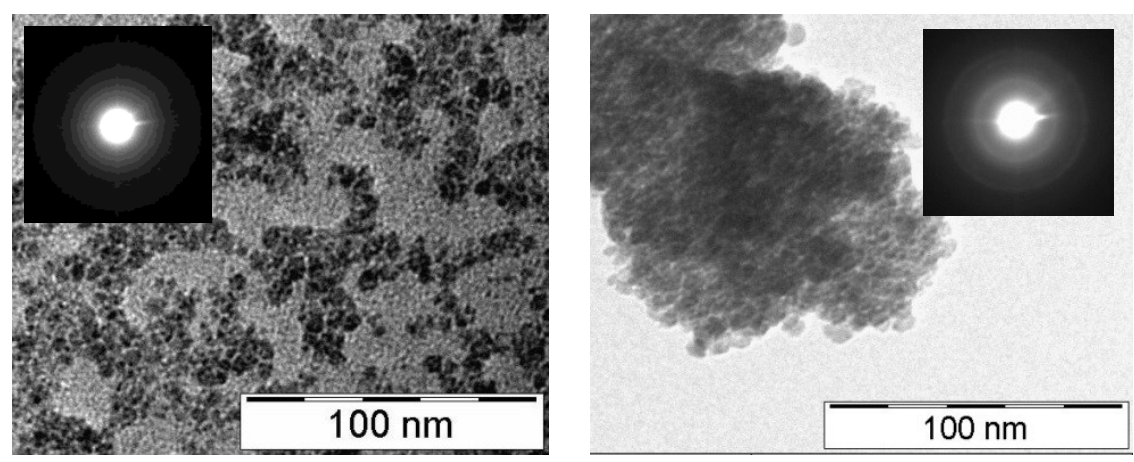

Figure 1. TEM images of sample NS1 (left) and NS2 (right). Note how the agglomeration morphology is influenced by the state of the samples being either colloidal suspension (NS1) or dried powder (NS2). At the same time, the characteristic particle size of the samples is similar, being $\sim 5 \mathrm{~nm}$ in both cases. Selected area diffraction patterns (overlaid of the corresponding TEM images) refer to similar crystal structures in the two cases. 


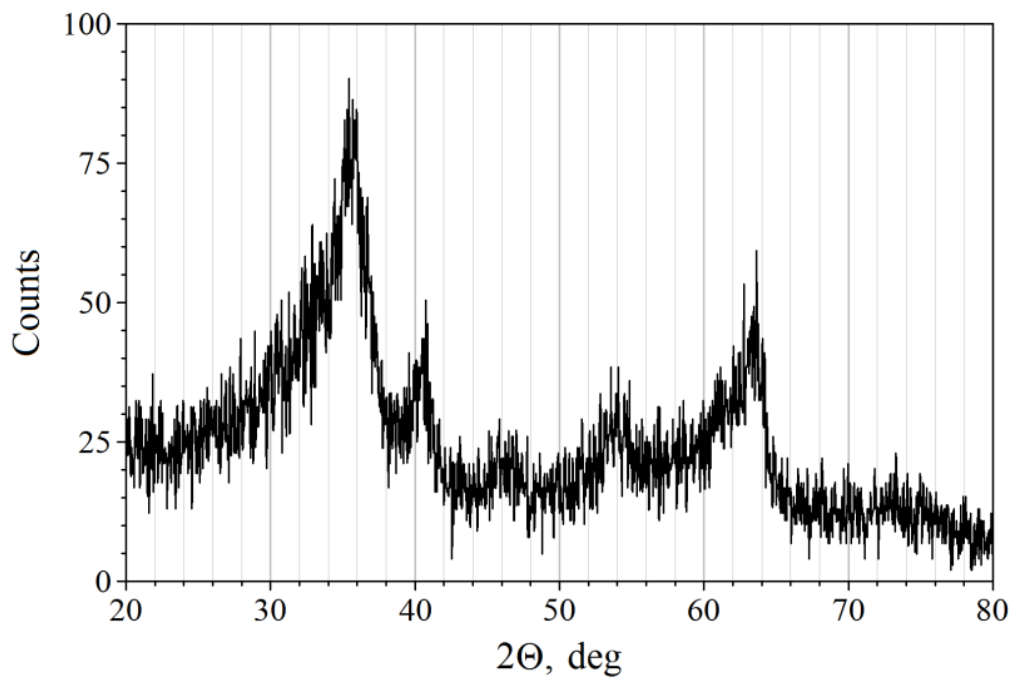

Figure 2. X-ray diffractogram of sample NS2. The observed pattern of broad reflections corresponds to that of 6-line ferrihydrite [5].

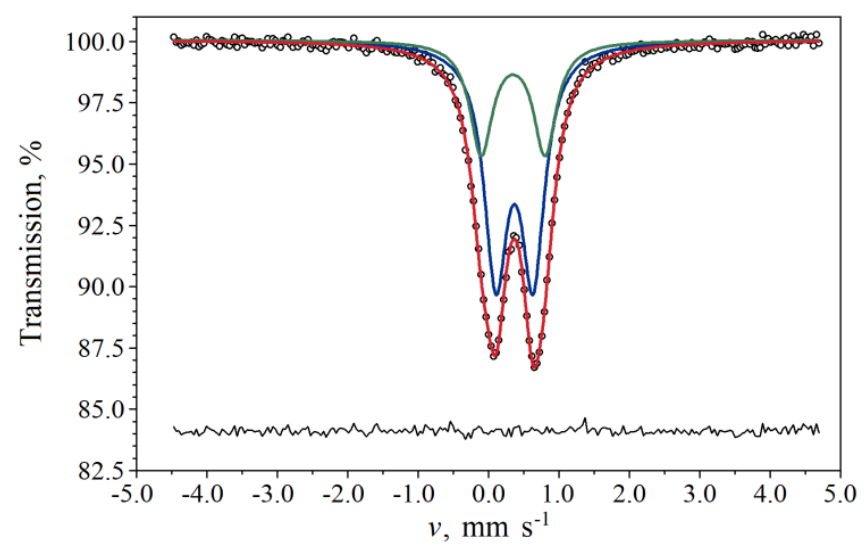

Figure 3. ${ }^{57} \mathrm{Fe}$ Mössbauer spectrum of sample NS2 at room temperature, decomposed into two Lorentzian doublets. See Table 1 for the corresponding Mössbauer parameters. The fit residual is shown below the spectrum.

Low temperature $(T \approx 80 \mathrm{~K}){ }^{57} \mathrm{Fe}$ Mössbauer spectra of NS1 (measured as frozen colloidal solution) and NS2 (powder) display considerable differences (Fig. 5). While NS2 is characterized with a spectrum that includes a well-developed sextet of absorption peaks without referring to the presence of a superparamagnetic component, separate doublet and sextet components can be observed in the case of NS1. Since, according to Fig. 2, the particle size and the crystal structure of the particles in the two samples are similar, the observed difference in the spectra may reflect the different agglomeration states of the particles in the two samples, with the apparently more prominent interparticle interactions ensuring an overall higher magnetic anisotropy energy barrier in the case of the powder sample NS2. 
Formal decomposition of the spectrum of the latter sample into two VBF sextets [7] having zero quadrupole shift and no coupling between $B_{\mathrm{hf}}$ and $\delta$ reveals a slight asymmetry of the full-blown sextet pattern, and yields an isomer shift of around $0.5 \mathrm{~mm} / \mathrm{s}$ for both components. In contrast, the sextet and doublet components in the spectrum of sample NS1 are characterized by a lower isomer shift of around $0.35 \mathrm{~mm} / \mathrm{s}$, evidencing a difference of puzzling nature whose roots need yet to be elucidated.
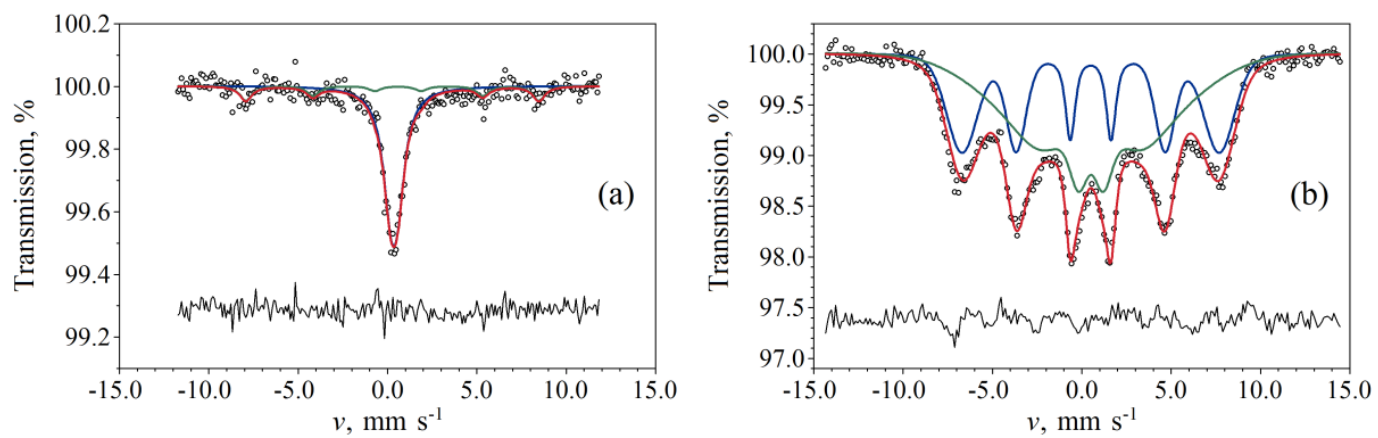

Figure $5 .{ }^{57} \mathrm{Fe}$ Mössbauer spectrum of (a) sample NS1 (suspension) and (b) sample NS2 (powder), both at $T \approx 80 \mathrm{~K}$. In each case the spectra were decomposed into two subcomponents. The corresponding residuals are shown below the spectra.

Administering sample NS1 (in the form of colloidal suspension) for one week to cucumber plants that were grown previously under iron deficient environment in hydroponics for two weeks resulted in a complete regeneration of the plant evidencing the promising beneficial effects of the nanoparticle suspension in alleviating the disadvantageous effects of iron deficiency.

Magnetite powder samples M2 and M4 were prepared with a particle size of $\sim 15 \mathrm{~nm}$ as confirmed by corresponding TEM images (Fig. 6). Strong agglomeration of particles is evidently present as expected for nanoparticles with a substantial magnetic moment and corresponding magnetic interparticle interaction.
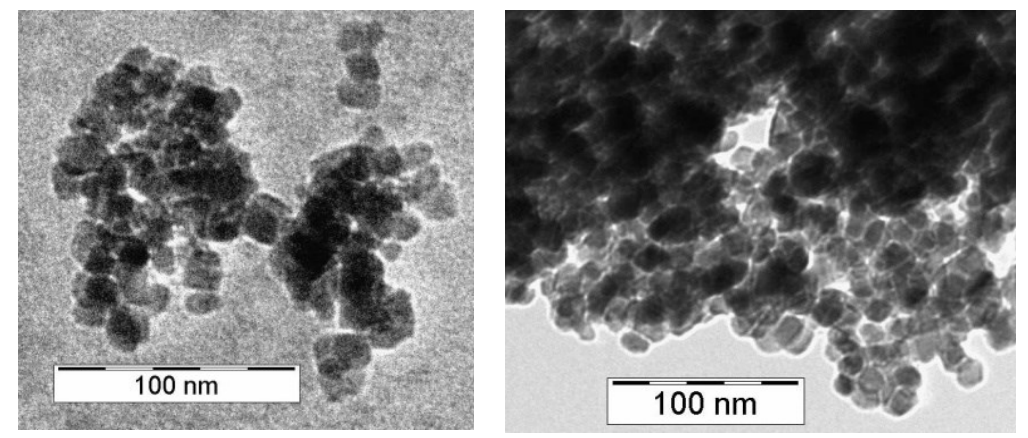

Figure 6. TEM images of magnetite sample M2 (left) and M4 (right). 
X-ray diffraction patterns of M2 and M4 (Fig. 7) could be indexed and fitted in accordance with the cubic spinel structure as expected for magnetite [8]. The crystallite size derived on the basis of the Scherrer equation conforms well with the characteristic particle size reflected by the TEM images (Fig. 6) suggesting that the nanoparticles were created in the form of single crystallites. The lattice parameters $(\sim 0.835 \mathrm{~nm}$ for M2 and $\sim 0.836 \mathrm{~nm}$ for M4 with a precision better than $0.0002 \mathrm{~nm}$ ) obtained from the fit of the diffractograms are below that typical for stoichiometric magnetite $(\sim 0.839 \mathrm{~nm}[8])$.This indicates that our samples may have formed with octahedral iron vacancies, and thereby resulting in samples with an overall composition of $\mathrm{Fe}_{3-x} \mathrm{O}_{4}$ where $x \in[0,1 / 3]$, known as nonstoichiometric magnetite [9].
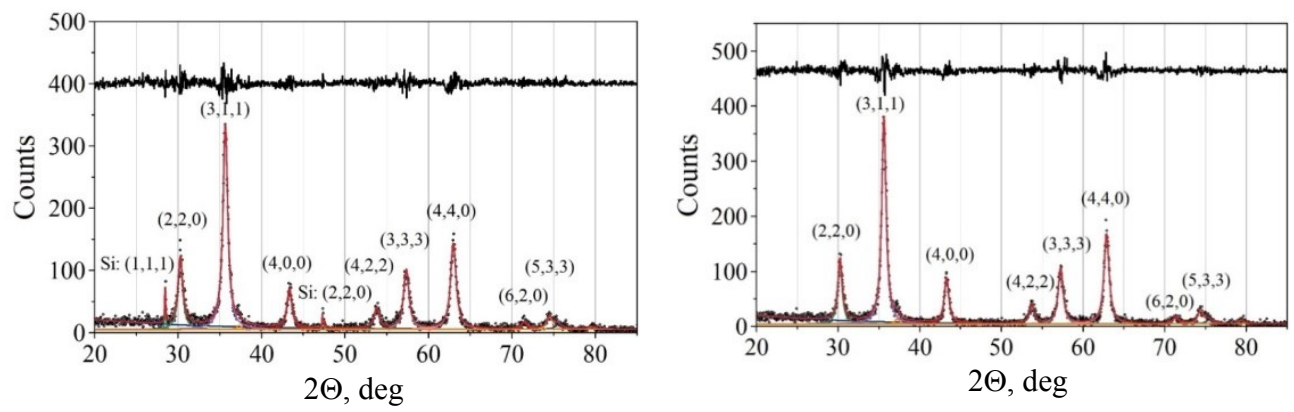

Figure 7. X-ray diffractograms of magnetite samples M2 (left) and M4 (right). In the diffractogram of sample M2 note the appearance of narrow Si peaks that were used to calibrate the $2 \Theta$ axis. Above the peaks the corresponding $(h, k, l)$ indexes are shown in accordance with the cubic spinel structure of magnetite. Solid curve indicates the envelope of the fit carried out with Lorentzian peaks having positions and widths in accordance with Bragg's law and the Scherrer equation. The residual of the fit is shown above the diffractograms.

The room temperature ${ }^{57} \mathrm{Fe}$ Mössbauer spectra of samples M2 and M4 display a sextet pattern with asymmetrically broadened peaks (Fig. 8). The observed peak broadening may be due to several reasons including hyperfine field distributions and relaxation effects. Hyperfine field distributions are expected to be present on the basis of cation non-stoichiometry (octahedral iron vacancies), whereas magnetic relaxation effects may occur due to the small particle size. As to a certain extent broadening due to relaxation effects may also be formally accounted for as if it was caused by a distribution in the hyperfine magnetic field values, we assumed that the spectra can be described by a certain number of VBF sextet components [7], and looked for the minimum number of spectral components that 
yields a mathematically acceptable fit with physically reasonable attributes. The Mössbauer spectra of a larger set of similar non-stoichiometric magnetite samples were fitted simultaneously with a set of physically reasonable constraints to be given in a detailed manner elsewhere. Here we present the results of this fit as regarding samples M2 and M4. Looking at Fig. 8 makes it clear that the spectra are very well described with $4 \mathrm{VBF}$ sextet components having quite different standard deviations of their underlying Gaussian hyperfine magnetic field $\left(B_{\mathrm{hf}}\right)$ distribution. In the case of these VBF sextets the isomer shift parameter $(\delta)$ was assumed to correlate in a linear manner with the hyperfine magnetic field, which gives rise to Gaussian isomer shift distributions associated with the respective HMF distributions. The $\delta$ - $B_{\mathrm{hf}}$ correlation coefficients were allowed to be different for the individual VBF components, but they were assumed to be independent from the sample.

One further constraint worth to mention is the fixing of the relative area fraction of the VBF sextet component (in the following 'Sextet 1') associated with the tetrahedral position in non-stoichiometric magnetite. Namely, this parameter varies with the non-stoichiometry level of magnetite only very mildly, taking on the values of $\sim 34.5 \%$ for stoichiometric magnetite and $\sim 37.5 \%$ for maghemite $\left(x=1 / 3\right.$ in $\left.\mathrm{Fe}_{3-x} \mathrm{O}_{4}\right)$. As the difference between these values is less than the typical statistical uncertainties observed for this relative area fraction when it is free to vary during the fit, it is reasonable to fix it to some selected value in the given range, for which latter we have chosen the extreme case of stoichiometric magnetite $(34.5 \%)$.

The cumulative hyperfine magnetic field and isomer shift distributions observed in the above way are depicted in Fig. 9 for both samples, and the corresponding main Mössbauer parameters are listed in Table 2. Whereas only moderate differences can be detected between the cumulative $B_{\mathrm{hf}}$ distributions, the associated cumulative isomer shift distributions (Fig. 9 (c) and (d)) are markedly different from each other in the samples M2 and M4. In the case of sample M4 a VBF sextet component (Sextet 4 in Table 2) is obtained whose mean isomer shift $(\sim 0.65 \mathrm{~mm} / \mathrm{s})$ is characteristic to that of $\mathrm{Fe}^{2.5+}$ at the octahedral site of magnetite [9]. However, this component is present with an area fraction of only $\sim 10 \%$, giving rise to further sextet components (Sextets 2 and 3 ) associated with octahedral iron. On the basis of its mean isomer shift $(\sim 0.39 \mathrm{~mm} / \mathrm{s})$ Sextet 2 
may be associated with $\mathrm{Fe}^{3+}$ ions at octahedral sites in $\mathrm{Fe}_{3-x} \mathrm{O}_{4}$ with $x>0$ [9]. With its isomer shift being intermediate between those of $\mathrm{Fe}^{3+}$ and $\mathrm{Fe}^{2.5+}$, Sextet 3 presumably indicates the presence of octahedral iron ions with intermediate oxidation states. In contrast with sample M4, in the Mössbauer spectrum of sample M2 only $\mathrm{Fe}^{3+}$ components are observed, referring to a more throughout oxidation of iron in this type of nanoparticles.
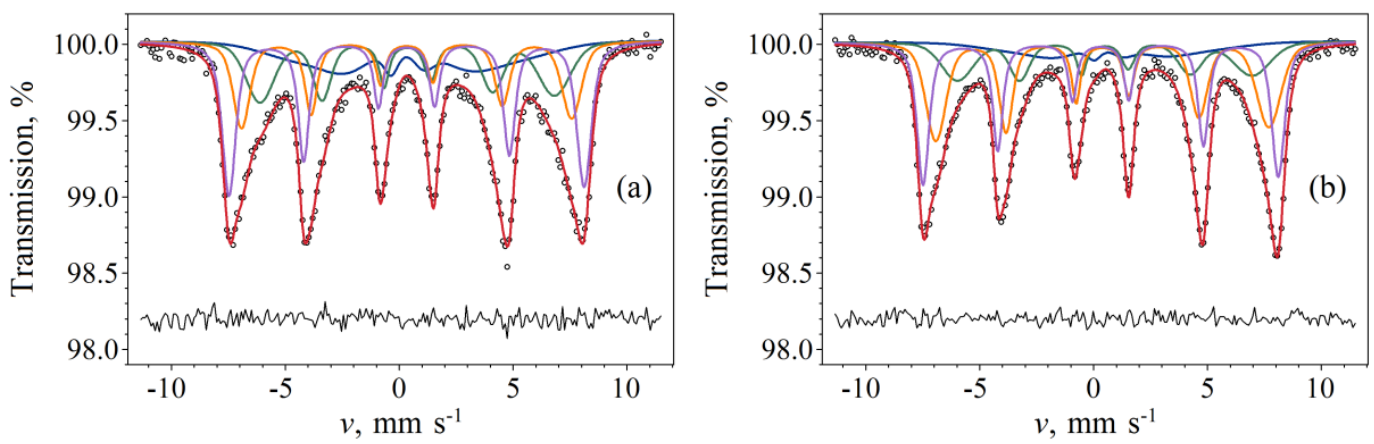

Figure 8. ${ }^{57} \mathrm{Fe}$ Mössbauer spectra of (a) M2 and (b) M4 magnetite samples recorded at room temperature. Both spectra were fitted to 4 VBF sextet components [7]. The residual of the fit is displayed below the spectra.
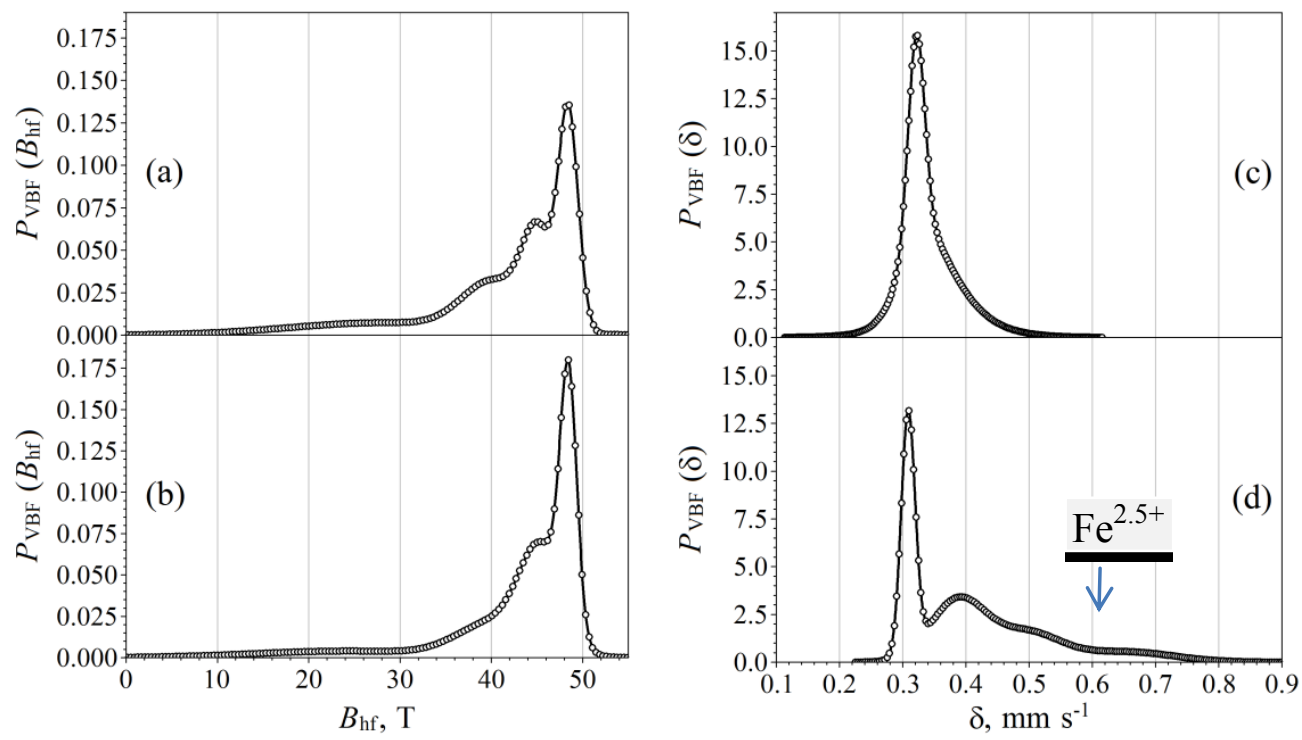

Figure 9.Cumulative ${ }^{57} \mathrm{Fe}$ hyperfine magnetic field and isomer shift distributions (in each case superposition of 4 Gaussian distributions underlying the corresponding VBF sextets of Figure 8) characterizing samples M2, via graphs (a) and (c), and M4, via graphs (b) and (d).

Table 2.The main Mössbauer parameters of the VBF sextet components used to describe the room temperature ${ }^{57} \mathrm{Fe}$ Mössbauer spectra (Figure 8) of samples M2 and M4. Numbers in parentheses 
give the statistical uncertainty ( 1 sigma) in the last digit of the corresponding values. (F) refers to a fixed parameter.

\begin{tabular}{|c|c|c|c|c|}
\hline & $\begin{array}{c}\text { Area fraction, } \\
\% \\
\end{array}$ & Mean $B_{\mathrm{hf}}, \mathrm{T}$ & Mean $\delta, \frac{\mathbf{m m}}{\mathbf{s}}$ & $\Gamma, \frac{\mathbf{m m}}{\mathbf{s}}$ \\
\hline \multicolumn{5}{|c|}{ Sample M2 } \\
\hline Sextet 1 & $34.5(\mathrm{~F})$ & $48.45(5)$ & $0.322(5)$ & \multirow{4}{*}{$0.50(1)$} \\
\hline Sextet 2 & $23(5)$ & $45.1(1)$ & $0.33(1)$ & \\
\hline Sextet 3 & $24(5)$ & $40.2(8)$ & $0.36(1)$ & \\
\hline Sextet 4 & $19(2)$ & $28(1)$ & $0.36(4)$ & \\
\hline \multicolumn{5}{|c|}{ Sample M4 } \\
\hline Sextet 1 & $34.5(F)$ & $48.46(4)$ & $0.309(5)$ & \multirow{4}{*}{$0.47(1)$} \\
\hline Sextet 2 & $35(4)$ & $45.4(1)$ & $0.39(1)$ & \\
\hline Sextet 3 & $20(4)$ & $40.2(8)$ & $0.50(2)$ & \\
\hline Sextet 4 & $10(1)$ & $24(1)$ & $0.65(8)$ & \\
\hline
\end{tabular}

The difference in the oxidation state of sample M2 and M4 shows that room temperature drying (as compared to $80^{\circ} \mathrm{C}$ ) and the presence of malic acid hampered oxidation of the magnetite nanoparticles.

\section{Conclusions}

We have successfully prepared iron(III) oxyhydroxide and (nonstoichiometric) magnetite nanoparticles with the aim to use them as possible nutrition source for plants. The iron(III)-oxyhydroxide nanoparticles investigated by XRD,TEM/SAED and Mössbauer spectroscopy proved to be 6-line ferrihydrite. It was experimentally verified that feeding plants with such nanoparticle suspension has a regenerative effect on iron deficient plants.

${ }^{57} \mathrm{Fe}$ Mössbauer spectra of magnetite nanoparticles prepared under different preparation conditions could be analyzed on the basis of a common model based on the superposition of four sextet components displaying Gaussian-shaped hyperfine magnetic field distributions. This showed that the magnetic nanoparticles are partially oxidized and may be described as $\mathrm{Fe}_{3-x} \mathrm{O}_{4}$. As learned from the isomer shifts obtained, the level of oxidation depended on the experimental conditions, namely, adding malic acid during the synthesis to 
stabilize nanoparticles and room temperature drying partially saved $\mathrm{Fe}^{2.5+}$ states characteristic to octahedral sites in bulk magnetite. Only $\mathrm{Fe}^{3+}$ states were identified when malic acid free synthesis was applied along with drying at $80^{\circ} \mathrm{C}$.

\section{Acknowledgements}

This work was supported by the Hungarian National Research, Development and Innovation Office - NKFIH (K115913 and K115784) and by the Coordenação de Aperfeiçoamento de Pessoal de Nível Superior (CAPES) (Project no. A127/2013).

\section{References}

[1] K. Kovács, E. Kuzmann, E. Tatár, A. Vértes, F. Fodor, Planta229 (2009) 271278.

[2] K. Kovács, J. Pechoušek, L. Machala, R. Zbořil, Z. Klencsár, Á. Solti, B. Tóth, B. Müller, H.D. Pham, Z. Kristóf, F. Fodor, Planta (2016) DOI:10.1007/s00425-016-2502-X

[3] V.J. Wade, A. Treffry, J.-P. Laulhère, E.R. Bauminger, M.I. Cleton, S. Mann, J.-F.Briat, P.M. Harrison, BiochimicaetBiophysicaActa1161 (1993) 91-96.

[4] Z. Klencsár, E. Kuzmann, A. Vértes, J. Radioanal. Nucl. Chem.210 (1996) $105-118$.

[5] D. Carta, M.F. Casula, A. Corrias, A. Falqui, G. Navarra, G. Pinna: Mat. Chem. Phys. 113 (2009) 349-355.

[6] E. Murad: Clay Minerals 14 (1979) 273-283.

[7] D.G. Rancourt, J.Y. Ping, Nucl. Instr. Meth. B 58 (1991) 85-97.

[8] C.A. Gorski, M. Scherer: American Mineralogist95(2010) 1017-1026.

[9] R.E. Vandenberghe, C.A. Barrero, G.M. da Costa, E. Van San, E. De Grave, Hyperfine Interactions 126 (2000) 247-259. 